DAMTP/94-81

\title{
Projective Quantum Spaces
}

\author{
U. MEYER \\ D.A.M.T.P., University of Cambridge \\ um102@amtp.cam.ac.uk
}

September 1994

\begin{abstract}
Associated to the standard $S U_{q}(n)$ R-matrices, we introduce quantum spheres $S_{q}^{2 n-1}$, projective quantum spaces $\mathbb{C P}_{q}^{n-1}$, and quantum Grassmann manifolds $G_{k}\left(\mathbb{C}_{q}^{n}\right)$. These algebras are shown to be homogeneous spaces of standard quantum groups and are also quantum principle bundles in the sense of T. Brzeziński and S. Majid [1].
\end{abstract}

\section{Introduction}

This paper gives a non-commutative generalisation of projective and Grassmann manifolds in the framework of quantum groups. Quantum groups are Hopf algebras with and additional structure (quasitriangularity or its dual version) and can be employed to construct a very powerful type of non-commutative geometry. For an introduction to quantum groups and their numerous other applications see the review article [4] or the textbooks $[7,2]$. We use the notation and standard constructions from [4].

The standard $S U_{q}(n)$ R-matrices were given in [9] as

$$
R_{n}=q^{-1 / n}\left(q \sum_{i} E_{i i} \otimes E_{i i}+\sum_{i \neq j} E_{i i} \otimes E_{j j}+\left(q-q^{-1}\right) \sum_{j>i} E_{i j} \otimes E_{j i}\right), q \in \mathbb{R} .
$$

Here $E_{i j}$ denote the elementary matrices with components $\left(E_{i j}\right)_{b}^{a}=\delta_{i}^{a} \delta_{b}^{j}$. These $\mathrm{R}$-matrices are invertible solutions of the $n$-dimensional quantum Yang-Baxter equation and are of real type, i.e. obey $\bar{R}_{n c d}^{a b}=R_{n}{ }^{d c} b a$. Furthermore, let $\varepsilon_{a_{1} \ldots a_{n}}=$ $\varepsilon^{a_{1} \ldots a_{n}}=(-q)^{l(\sigma)}$, where $l(\sigma)$ is the length of the permutation $\sigma(1, \ldots, n)=$ $\left(a_{1}, \ldots, a_{n}\right)$, and define quantum determinants as

$$
\operatorname{det}_{q, n}=\nu_{n}^{-1} \varepsilon_{a_{1} \ldots a_{n}} t_{b_{1}}^{a_{1}} \ldots t_{b_{n}}^{a_{n}} \varepsilon^{b_{n} \ldots b_{1}} .
$$

The normalisation factor is $\nu_{n}=\varepsilon_{a_{1} \ldots a_{n}} \varepsilon^{a_{n} \ldots a_{1}}$.

In terms of these data, the quantum groups $S U_{q}(n)$ were defined in [9] as the quotients $A\left(R_{n}\right) / \operatorname{det}_{q, n}=1$. This algebra has well-known antipode $S[9$, Theorem 4] 
and $*$-structure $t_{b}^{a *}=S t^{b}{ }_{a}$ such that $t g t^{\dagger}=g$ where $t^{\dagger}=S t$ and $g_{b}^{a}=q^{2(a-n)-1} \delta_{b}^{a}$. We also define quantum groups $U_{q}(n)$ as the quotients of the algebras $A\left(R_{n}\right)$ by the relations $\operatorname{det}_{q, n} \operatorname{det}_{q, n}^{*}=1$. For $n=1$, one recovers the undeformed algebra of complex polynomial functions on $U(1)$, i.e. $U_{q}(1)$ is the associative $\mathbb{C}$-algebra generated by elements $z$ and $z^{*}$ such that $z^{*}=z^{-1}$.

These quantum groups act covariantly on the algebra of quantum vectors $V\left(R_{n}\right)$ with generators $v^{i}, i \in\{1, \ldots, n\}$ and relations $v^{i} v^{j}=\lambda_{n}^{-1} R_{n k l}{ }^{i j} v^{l} v^{k}$. Here the normalisation factors are $\lambda_{n}=q^{1-1 / n}$. The coaction is given by $v^{i} \mapsto v^{k} \otimes t^{\dagger i}$. Similarly, the algebra of quantum covectors $V^{*}\left(R_{n}\right)$ has generators $x_{i}$, relations $x_{i} x_{j}=x_{l} x_{k} \lambda_{n}^{-1} R_{n}{ }_{i j}^{k l}$, and coaction $x_{i} \mapsto x_{k} \otimes t_{i}^{k}$. In terms of these algebras, we define $\mathbb{C}_{q}^{n}=V\left(R_{n}\right) \underline{\otimes} V^{*}\left(R_{n}\right)$ as their braided tensor product (See [5] for an introduction to braided geometry). For the braiding between $V\left(R_{n}\right)$ and $V^{*}\left(R_{n}\right)$ we do not take the braiding induced by the coaction by $S U_{q}(n)$ as given in [6], but $\Psi\left(x_{i} \otimes v^{j}\right)=v^{k} \otimes x_{l} \lambda_{n} \widetilde{R}_{n}^{l j}{ }_{i k}$, which differs from the $S U_{q}(n)$-braiding by a factor $\lambda_{n}$, i.e. is the braiding induced by a suitable dilatonic extension of $S_{q}(n)$. We equip this algebra with the $*$-structure $\left(1 \otimes x_{a}\right)^{*}=v^{a} \otimes 1$ and define the quantum norm $N_{q, n}=v^{a} \otimes g_{a}^{b} x_{b}$ where $g_{a}^{b}=\lambda_{n} \widetilde{R}_{n}^{b c}$ ca. The matrix $\widetilde{R}$ is defined as $\left(\left(R^{t_{2}}\right)^{-1}\right)^{t_{2}}$, where $t_{2}$ denotes transposition in the second tensor component. Since $R_{n}$ is of real type, $g$ is symmetric and the quantum norm is real. It is known from [6, Theorem 3.6] that for any invertible solution $R$ of the quantum Yang-Baxter equation the quantum norm is also central. For the R-matrices (11), this general construction reproduces the q-metric introduced above: one finds

$$
\widetilde{R}_{n}=q^{1 / n}\left(q^{-1} \sum_{i} E_{i i} \otimes E_{i i}+\sum_{i \neq j} E_{i i} \otimes E_{j j}-\left(q-q^{-1}\right) \sum_{j>i} q^{-2(j-i)} E_{i j} \otimes E_{j i}\right),
$$

and hence $g_{b}^{a}=q^{2(a-n)-1} \delta_{a}^{b}$.

We will use the algebras $\mathbb{C}_{q}^{n}$ to define quantum spheres and complex projective quantum spaces. These algebras are then recovered as base spaces of quantum principle bundles with $U_{q}(n)$ and $S U_{q}(n)$ as structure groups. The appropriate definition of a quantum principle bundle was given by T. Brzeziński and S. Majid in [1, Definition 4.1]: an algebra $P$ is a quantum principle bundle with structure quantum group $A$ and base $B$ if (i) $A$ is a Hopf algebra, (ii) $P$ is a left $A$-comodule algebra with coaction $\beta: P \rightarrow A \otimes P$, and (iii) $B=P^{A}=\{u \in P: \beta(u)=1 \otimes u\}$ is the subalgebra of $A$-invariant elements of $P$. Additionally, there is a freeness and an exactness condition. However, if $B$ is a quantum homogeneous space, i.e. if there is a Hopf algebra surjection $\pi: P \rightarrow A$ between Hopf algebras $A$ and $P$ and if additionally

$$
\operatorname{ker} \pi \subset \cdot\left(\left.\operatorname{ker} \pi\right|_{B} \otimes P\right)
$$


then the freeness and exactness condition are automatically satisfied [1, Lemma 5.2] and the quantum homogeneous space $B=P^{A}$ is a quantum principle bundle. In this case the map $\pi$ is called exact.

\section{Quantum spheres $S_{q}^{2 n-1}$}

Quantum spheres are quotients of $\mathbb{C}_{q}^{n}$ and feature as base spaces of various quantum principle bundles.

DEFINITION 2.1 Quantum spheres $S_{q}^{2 n-1}$ are defined as the quotients $\mathbb{C}_{q}^{n} / N_{q, n}=1$.

Due to the relation $t g t^{\dagger}=g$, the quantum spheres $S_{q}^{2 n-1}$ are covariantly coacted upon by both $U_{q}(n)$ and $S U_{q}(n)$. For the special cases where $n$ equals 1 or 2 , one finds:

EXAMPLE $2.2 S_{q}^{1} \cong U_{q}(1)$ and $S_{q}^{3} \cong S U_{q}(2)$ as $*$-algebras.

Proof. The first isomorphism is trivial and the second one is easily established by rescaling of the generators of $S_{q}^{3}$. This isomorphism only holds if one introduces the normalisation factor $\lambda_{2}$ in the definition of the braiding in $\mathbb{C}_{q}^{2}$.

PROPOSITION 2.3 There are $n$ *-algebra injections $\imath_{m}: S_{q}^{2 n-1} \hookrightarrow U_{q}(n)$.

Proof. In this proof, there is no summation over the variable $m$. Define the maps $\imath_{m}$ by $x_{a} \mapsto t_{a}^{m}$. By inspection of (11) one finds $R_{n}^{m m}=q^{1-1 / n} \delta_{c}^{m} \delta_{d}^{m}$ and hence the relations in $A\left(R_{n}\right)$ imply $q^{1-1 / n} t_{a}^{m} t_{b}^{m}=R_{n}^{m m_{d}} t_{a}^{c} t_{b}^{d}=t_{d}^{m} t_{c}^{m} R_{n a b}^{c d}$, i.e. the generators $t_{a}^{m}, a \in\{1, \ldots, n\}$ obey the relations of $V^{*}\left(R_{n}\right)$. Since $R_{n}$ is of real type, the generators $t^{\dagger a}{ }_{m}, a \in\{1, \ldots, n\}$ then obey the $V\left(R_{n}\right)$-relations. Next note that the relations in $A\left(R_{n}\right)$ can be written as $t^{\dagger a}{ }_{b} R_{n}{ }^{c b}{ }_{d e} t_{g}^{d}=t_{d}^{c} R_{n}{ }_{g b}{ }^{\dagger b} t_{e}$ and hence with $R_{n m g}^{a m}=q^{1-1 / n} \delta_{m}^{a} \delta_{g}^{m}$ one finds $t_{i}^{m} t^{\dagger j}{ }_{m}=\widetilde{R}_{n}{ }_{i f}{ }_{i f}^{\dagger f} t_{b}^{\dagger f} R_{n}{ }^{m b}{ }^{c} t_{d}^{c}=q^{1-1 / n} \widetilde{R}_{n}{ }^{d j}{ }_{i f} t_{m}^{\dagger f} t_{d}^{m}$. Thus the relations between $t_{i}^{m}$ and $t_{m}^{\dagger j}$ reproduce the braiding between $V\left(R_{n}\right)$ and $V^{*}\left(R_{n}\right)$ given above. This is the place where one needs the normalisation factor $\lambda_{n}$ in the definition of the braiding. Finally note, that $t^{\dagger a}{ }_{m} g_{a}^{b} t_{b}^{m}=\lambda_{n} t^{\dagger a}{ }_{m} \widetilde{R}_{n}{ }^{b i}{ }^{i} t_{b}^{m}=$ $t_{i}^{m} t^{\dagger i}=1$. Hence the maps $\imath_{m}$ are $*$-algebra injections.

For the construction of quantum principle bundles over these quantum spheres we need the following lemma: 
LEMMA 2.4 There are surjective *-Hopf algebra morphisms

$$
\begin{aligned}
& \alpha_{n}: U_{q}(n) \rightarrow U_{q}(n-1) \\
& \gamma_{n}: S U_{q}(n) \rightarrow S U_{q}(n-1) \text {. }
\end{aligned}
$$

Proof. By inspection of (四) one finds that for $i, j>1, R_{n}{ }^{i j}{ }_{k l}=R_{n-1(k-1)(l-1)}{ }^{(i-1)(j-1)}$ and also that $R_{n i j}^{11}=q^{1-1 / n} \delta_{i}^{1} \delta_{j}^{1}$. Thus the elements $t_{j}^{i} ; i, j \in\{2, \ldots, n\}$ of $A\left(R_{n}\right)$ obey the relations of $A\left(R_{n-1}\right)$ and the quotient maps by the ideal generated by $t_{1}^{1}=1$ and $t_{i}^{1}=t_{1}^{i}=0, i \in\{2, \ldots, n\}$ are surjections $A\left(R_{n}\right) \rightarrow A\left(R_{n-1}\right)$. These quotient maps also have the property $\operatorname{det}_{q, n} \mapsto \operatorname{det}_{q, n-1}$ and thus descend to surjective *-Hopf algebra maps $U_{q}(n) \rightarrow U_{q}(n-1)$ and $S U_{q}(n) \rightarrow S U_{q}(n-1)$, which we denote by $\alpha_{n}$ and $\gamma_{n}$, respectively.

THEOREM 2.5 The Hopf algebra $U_{q}(n)$ is a quantum principle bundle with base $S_{q}^{2 n-1}$ and structure quantum group $U_{q}(n-1)$ :

$$
S_{q}^{2 n-1} \cong U_{q}(n)^{U_{q}(n-1)}
$$

The left covariant coaction of $U_{q}(n-1)$ on $U_{q}(n)$ is given by $\left(\alpha_{n} \otimes i d\right) \circ \Delta$ in terms of the morphism $\alpha_{n}$ from lemma 2.4.

Proof. The subalgebra of $U_{q}(n-1)$-invariant elements of $U_{q}(n)$ is generated by $t_{i}^{1}, i \in\{1, \ldots, n\}$ and their conjugates. Proposition 2.3 then implies (3). Since $\operatorname{ker} \alpha_{n}=\left.\operatorname{ker} \alpha_{n}\right|_{S_{q}^{2 n-1}}$ the maps $\alpha_{n}$ are exact in the sense of (2) and the quantum homogeneous space is a quantum principle bundle.

One can repeat this construction with the maps $\gamma_{n}: S U_{q}(n) \rightarrow S U_{q}(n)$ from lemma 2.4 to establish that $S U_{q}(n)$ is also a quantum principle bundle over a quantum sphere:

THEOREM 2.6 The Hopf algebra $S U_{q}(n)$ is a quantum principle bundle with base $S_{q}^{2 n-1}$ and structure quantum group $S U_{q}(n-1)$ :

$$
S_{q}^{2 n-1} \cong S U_{q}(n)^{S U_{q}(n-1)}
$$

The left covariant coaction of $S U_{q}(n-1)$ on $S U_{q}(n)$ is given by $\left(\gamma_{n} \otimes i d\right) \circ \Delta$.

\section{Complex projective quantum spaces}

We now turn our attention to complex projective spaces which are defined as $U_{q}(1)$-covariant subalgebras of quantum spheres $S_{q}^{2 n-1}$. For this purpose note 
that by virtue of lemma 2.4, the right covariant $*$-coaction $\beta: \mathbb{C}_{q}^{n} \rightarrow \mathbb{C}_{q}^{n} \otimes U_{q}(n)$ descends to a covariant $U_{q}(1)$-coaction defined as $\left(i d \otimes\left(\alpha_{2} \circ \alpha_{3} \circ \ldots \circ \alpha_{n}\right)\right) \circ \beta$. This coaction is given explicitly by $x^{i} \mapsto x^{i} \otimes z$ and also descends to a covariant *-coaction on $S_{q}^{2 n-1}$.

DEFINITION 3.1 Complex projective quantum space $\mathbb{C P}_{q}^{n}$ is defined as

$$
\mathbb{C P}_{q}^{n-1}=\left(S_{q}^{2 n-1}\right)^{U_{q}(1)},
$$

the subalgebra of $U_{q}(1)$-invariant elements of the quantum sphere $S_{q}^{2 n-1}$.

Since quantum projective spaces are merely subalgebras of quantum spheres $S_{q}^{2 n-1}$, they are also covariantly coacted upon by $S U_{q}(n)$ and $U_{q}(n)$. Note also that their relations are independent of the normalisation factor $\lambda_{n}$ which we introduced in the definition of $\mathbb{C}_{q}^{n}$, i.e. at this stage one could work with the standard $S U_{q}(n)$ braiding.

For the construction of quantum principle bundles on these complex projective quantum spaces we need the following lemma:

LEMMA 3.2 There are a surjective *-Hopf algebra morphisms

$$
\delta_{n}: S U_{q}(n) \rightarrow U_{q}(n-1) .
$$

Proof. The proof is similar to the proof of lemma 2.4. The maps $\delta_{n}$ are defined like $\beta_{n}$ with the only difference that $\delta_{n}\left(t_{1}^{1}\right)=\left(\operatorname{det}_{q, n-1}\right)^{*}$.

THEOREM 3.3 The Hopf algebra $S U_{q}(n)$ is a quantum principle bundle with base $\mathbb{C P}_{q}^{n-1}$ and structure quantum group $U_{q}(n-1)$ :

$$
\mathbb{C P}_{q}^{n-1} \cong S U_{q}(n)^{U_{q}(n-1)}
$$

The coaction of $U_{q}(n-1)$ on $S U_{q}(n)$ is given by $\left(\delta_{n} \otimes i d\right) \circ \Delta$.

Proof. In this case, invariant generators are products $t^{\dagger i}{ }_{1} t_{j}^{1}, i, j \in\{1, \ldots, n\}$ which generate the subalgebra of $U_{q}(1)$-invariant elements of $S_{q}^{2 n-1}$, i.e. $\mathbb{C P}_{q}^{n-1}$. The kernel of $\delta_{n}$ is generated by $t^{i}{ }_{1}$ and $t_{i}^{1}$, where $i \in\{2, \ldots n\}$ and the kernel of the restricted map $\left.\operatorname{ker} \delta_{n}\right|_{\mathbb{C P}_{q}^{n-1}}$ has generators $t_{i}^{1} t^{\dagger j}{ }_{1}$ where either $i$ or $j$ is larger that 1. The exactness of $\delta_{n}$ is then established by the trivial observation $t^{\dagger i}{ }_{1}=\cdot\left(t^{\dagger i}{ }_{1} t_{j}^{1} \otimes t^{\dagger j}{ }_{1}\right)$ and the relation $t_{i}^{1}=t_{i}^{1} t^{\dagger j}{ }_{1} g_{j}^{k} t_{k}^{1}=\lambda_{n} \widetilde{R}_{n}{ }_{i f}{ }_{i f} t^{\dagger f}{ }_{1} t_{d} g^{k}{ }_{j} t_{k}{ }_{k}=$ 
$\cdot\left(t^{\dagger f}{ }_{1} t_{d}^{1} \otimes \lambda_{n} \widetilde{R}_{n}{ }^{d j}{ }_{i f} g_{j}^{k} t_{k}^{1}\right)$. Here we used the formulae from the proof of proposition 2.3 and the fact that for $i \neq 1, \widetilde{R}_{n}^{d j}$ if is non-zero only if $d \neq 1$.

As a corollary of theorem 2.5 one finds with definition 3.1 that complex projective quantum spaces $\mathbb{C P}_{q}^{n}$ can also be regarded as homogeneous quantum spaces of $U_{q}(n)$ :

THEOREM 3.4 The Hopf algebra $U_{q}(n)$ is a quantum principle bundle with base $\mathbb{C P}_{q}^{n}$ and structure quantum group $U_{q}(1) \otimes U_{q}(n-1)$ :

$$
\mathbb{C P}_{q}^{n} \cong U_{q}(n)^{U_{q}(1) \otimes U_{q}(n-1)}
$$

The covariant coaction by $U_{q}(1) \otimes U_{q}(n-1)$ is given explicitly in the next section, where we generalise this construction to quantum Grassmann manifolds.

We now restrict our attention to the case of $n=2$. In this case, the algebra $S U_{q}(2)^{U_{q}(1)}$ is a deformation of the two-sphere and was already discussed by $\mathrm{P}$. Podles̀ in [8], where it was denoted by $S_{q}^{2}$. As a corollary of theorem 3.3 we hence find:

COROLLARY 3.5 Projective quantum space $\mathbb{C P}_{q}^{1}$ is isomorphic to Podles̀'s quantum sphere $S_{q}^{2}$.

The $n=2$ case is also in the undeformed case quite remarkable since all spaces involved are spheres: $S U(2) \cong S^{3}, U(1) \cong S^{1}$ and $\mathbb{C P}^{1} \cong S^{2}$. The corresponding principle bundle is the celebrated Hopf fibration of the three sphere. In the quantum case one finds with example 2.2 and theorem 3.3 :

COROLLARY 3.6 ( Hopf fibration of the quantum 3-sphere) There is a quantum principle bundle

$$
S_{q}^{2} \cong\left(S_{q}^{3}\right)^{S_{q}^{1}}
$$

The quantum homogeneous space (4) was already discussed in [11], although not as quantum principle bundles, an appropriate definition of which was only given a few years later.

\section{Quantum Grassmann manifolds}

The construction encountered in theorem 3.4 can be generalised to give a definition of quantum Grassmann manifolds as quantum principle bundles. For this purpose, we prove the following lemma: 
LEMMA 4.1 There are surjective *-Hopf algebra morphisms

$$
\alpha_{k, n}: U_{q}(n) \rightarrow U_{q}(k) \otimes U_{q}(n-k)
$$

Proof. Note that for $i, j<n, R_{n k l}^{i j}=R_{n-1 k l}^{i j}$, i.e. the elements $t_{j}^{i} ; i, j \in$ $\{1, \ldots, n-1\}$ of $A\left(R_{n}\right)$ obey the relations of $A\left(R_{n-1}\right)$. Similar to lemma 2.4 one can thus define surjective $*$-Hopf algebra morphisms $\alpha_{n}^{\prime}: U_{q}(n) \rightarrow U_{q}(n-1)$ by dividing by $t_{n}^{n}=1$ (no summation!) and $t_{i}^{n}=t_{n}^{i}=0, i \in\{1, \ldots, n-1\}$. The maps $\left(\left(\alpha_{k+1} \circ \alpha_{k+2} \circ \ldots \circ \alpha_{n}\right) \otimes\left(\alpha_{n-k+1}^{\prime} \circ \ldots \circ \alpha_{n}^{\prime}\right)\right) \circ \Delta$ are then the required $*$-Hopf algebra maps $\alpha_{k, n}$

The covariant coaction by $U_{q}(1) \otimes U_{q}(n-1)$ on $U_{q}(n)$ from theorem 3.4 is given by $\left(\alpha_{1, n} \otimes i d\right) \circ \Delta$. However, the real merit of this lemma is that it leads to a straightforward generalisation from quantum projective spaces to quantum Grassmann manifolds:

DEFINITION 4.2 Quantum Grassmann manifolds are defined as the quantum principle bundles

$$
G_{k}\left(\mathbb{C}_{q}^{n}\right)=U_{q}(n)^{U_{q}(k) \otimes U_{q}(n-k)}
$$

where the covariant coaction is given by $\left(\alpha_{k, n} \otimes i d\right) \circ \Delta$. This definition includes the case of projective quantum spaces as $G_{1}\left(\mathbb{C}_{q}^{n}\right)=\mathbb{C P}_{q}^{n}$.

A useful set of generators of $G_{k}\left(\mathbb{C}_{q}^{n}\right)$ is given by the elements

$$
\left(\varepsilon_{c_{1} \ldots c_{k}} t_{a_{1}}^{c_{1}} \ldots t_{a_{k}}^{c_{k}}\right)^{*}\left(\varepsilon_{d_{1} \ldots d_{k}} t_{b_{1}}^{d_{1}} \ldots t_{b_{k}}^{d_{k}}\right),
$$

where $c_{i}, d_{i} \in\{1, \ldots, k\}$ and $a_{i}, b_{i} \in\{1, \ldots, n\}$.

An earlier paper by E. Taft and J. Towber [10] (see also [3]) presented a related approach to quantum Grassmann manifolds and studied the algebra generated by elements $\varepsilon_{d_{1} \ldots d_{k}} t_{b_{1}}^{d_{1}} \ldots t_{b_{k}}^{d_{k}}$, where the $t_{b}^{a}$ are the generators of $A\left(R_{n}\right)$ and not of the quotient $U_{q}(n)$. Thus a quotient of a subalgebra of the algebra from [10, Proposition 3.4] is the quantum Grassmann manifold $G_{k}\left(\mathbb{C}_{q}^{n}\right)$. 


\section{References}

1. T. Brzezinski and S. Majid, Comm. Math. Phys. 157, 591 (1993).

2. V. Chari and A. Pressley, A Guide to Quantum Groups, C.U.P., 1994.

3. V. Lakshmibai and N. Reshetikhin, C. R. Acad. Sci. Paris I 313 (3), 121 (1991)

4. S. Majid, Int. J. Mod. Phys. A 5, 1 (1990).

5. S. Majid, in: M.-L. Ge and H. J. de Vega, Proc. 5th Nankai Workshop, World Scientific, 1992.

6. S. Majid, J. Math. Phys. 34, 1176 (1993).

7. S. Majid, Foundations of Quantum Group Theory, C.U.P. (to be published)

8. P. Podles, Lett. Math. Phys. 14, 193 (1987).

9. N. Reshetikhin, L. Takhtadzhyan, and L. Faddeev, Leningrad Math. J. 1 (1), 193 (1990).

10. E. Taft and J. Towber, J. Algebra 142, 1 (1991).

11. S. L. Woronowicz, Publ. RIMS 23, 117 (1987). 